\title{
Blastic Transformation of a Mantle Cell Lymphoma Presenting as an Enlarging Unilateral Orbital Mass
}

\author{
Matthew A. De Niear ${ }^{a} \quad$ John P. Greer ${ }^{b}$ Adam Seegmiller ${ }^{c}$ Louise A. Mawn ${ }^{\text {d, e }}$ \\ ${ }^{a}$ Medical Scientist Training Program, Vanderbilt University School of Medicine, Nashville, TN, USA; ${ }^{b}$ Division \\ of Hematology/Oncology, Department of Medicine, Vanderbilt University Medical Center, Nashville, TN, USA; \\ 'Department of Pathology, Microbiology, and Immunology, Vanderbilt University Medical Center, Nashville, TN, \\ USA; ${ }^{d}$ Department of Ophthalmology and Visual Sciences, Vanderbilt Eye Institute, Vanderbilt University Medical \\ Center, Nashville, TN, USA; ${ }^{e}$ Department of Neurological Surgery, Vanderbilt University Medical Center, Nashville, \\ TN, USA
}

\section{Established Facts}

- Mantle cell lymphoma (MCL) is an uncommon form of non-Hodgkin lymphoma that may affect the orbital and ocular adnexal regions.

- The blastoid variant of MCL may develop de novo or, in rare cases, following transformation from classical MCL to the blastoid variant of MCL.

\section{Novel Insights}

- New involvement of the orbit and ocular adnexa by MCL cannot be presumed to exhibit the same characteristics as MCL at other sites.

- Blastic transformation of MCL may occur within the orbit and biopsy samples may contain both classical MCL and blastoid MCL components.

\section{Keywords}

Mantle cell lymphoma · Orbital lymphoma · Blastic transformation - Ocular adnexa - Blastoid variant of mantle cell lymphoma

\section{Abstract \\ Mantle cell lymphoma (MCL) is an uncommon form of non- Hodgkin lymphoma predominantly affecting male individu-}

als of advanced age. Approximately 1-9\% of cases of lymphoma affecting the orbital and ocular adnexal regions are attributed to MCL. We describe the case of a 65-year-old man with a 24-year history of MCL with initial remission followed by multiple relapses who presented with acute-onset binocular diplopia and proptosis of the left eye. Subsequent imaging demonstrated a new left superior orbital mass. Biopsy of the mass revealed two clonally related, yet distinct cellular components demonstrating the classical mantle cell mor-

\section{KARGER}

(C) 2018 S. Karger AG, Basel

E-Mail karger@karger.com

www.karger.com/oop
Louise A. Mawn, MD, FACS

Vanderbilt Eye Institute

2311 Pierce Ave

Nashville, TN 37232 (USA)

E-Mail Louise.A.Mawn@vanderbilt.edu 
phology and large cells that appeared to have transformed to the blastoid variant of MCL. Transformation of classical $M C L$ to the blastoid variant of $M C L$ is rare, with few reports in the literature. The blastoid variant of $M C L$ tends to be aggressive and associated with a poor prognosis. The case we describe represents perhaps the first report of MCL transformation observed in the orbit.

(C) 2018 S. Karger AG, Basel

\section{Introduction}

Mantle cell lymphoma (MCL) is a subtype of nonHodgkin lymphoma with distinct clinical and molecular features and comprises approximately 3-6\% of all cases of non-Hodgkin lymphoma [1]. In the United States, recent estimates suggest the age-adjusted incidence of MCL to be approximately $0.91-1.01$ per 100,000 persons [2]. MCL occurs predominantly in males (the relative risk for males is estimated between 2.65 and 3.00 [2]) and with more advanced age (median age in the 60s) [2-6]. MCL is typically aggressive and many patients often present with disseminated disease [7]. Extranodal involvement in MCL is common with sites of reported involvement including the peripheral blood, bone marrow, and gastrointestinal tract as well as other less frequently reported sites such as the orbit $[3,8]$. Usually, MCL exhibits short responses to treatments and frequent relapses [7], with median overall survival estimates of $4-5$ years $[2,4]$ (although there are some cases of MCL with a more indolent form of the disease and long survival) [7].

MCL is associated with the $\mathrm{t}(11 ; 14)(\mathrm{q} 12 ; \mathrm{q} 32)$ translocation in which the BCL1 (CCND1) gene (11q13) that normally codes for the protein cyclin D1 is positioned near the immunoglobulin heavy-chain (IGH) gene (14q32) leading to an upregulation of BCL1 and overproduction of cyclin D1 [9]. Although immunohistochemistry staining for cyclin D1 is typically positive for most cases of MCL, a small portion of cases of MCL may not show positive cyclin D1 and are believed to be related to overexpression of cyclin D2 or cyclin D3 [4] and associated with translocations involving CCND2 and CCND3 [1]. SOX11, a transcription factor, has been suggested to be helpful in the identification of cyclin D1-negative MCL as SOX11 mRNA and nuclear protein expression are reported to be relatively specific markers for MCL [10].

MCL cells typically show expression of B-cell-associated antigens CD20, CD22, CD79, and the T-cell-associated antigen $\mathrm{CD} 5$, and do not usually express CD23, CD10, or BCL6 [1, 7]. Classical MCL has been described morphologically as small to medium-sized lymphocytes with irregularly shaped nuclei and inconspicuous nucleoli [7]. Morphologic variants of MCL include the blastoid form, which is typically found to arise de novo although rare transformations of the classical form to the blastoid variant have been reported [11]. The blastoid variant has been described morphologically as medium-sized cells with scant and indistinct cytoplasm and finely dispersed chromatin with barely distinguished nucleoli although some cases may also include large cells with centroblastic morphology, round nuclei, fine chromatin, prominent nucleoli, and a moderate amount of basophilic cytoplasm [12]. The blastoid variant of MCL is aggressive [13] and is typically associated with poor outcomes [11, 14].

MCL involving the ocular regions is rare relative to other lymphomas involving the ocular regions $[15,16]$. We present the case of a patient with MCL involving the orbit that was found to exhibit transformation from classical MCL to the blastoid variant of MCL in the same specimen.

\section{Case Report}

A 65-year-old male patient with a 24-year history of MCL presented with acute onset of left eye proptosis, blurred near vision, and binocular diplopia that began following a 3- to 4-day history of his eye feeling "different," without pain. The acute onset of the patient's proptosis was confirmed by the patient's wife. The patient's MCL had previously exhibited a relatively indolent course, with involvement at multiple sites and a variable response to multiple forms of chemotherapy, including treatments with chlorambucil; fludarabine; cyclophosphamide, hydroxydoxorubicin, oncovin, prednisone, rituximab (CHOP-R); bortezomib-R; R-ifosfamide, carboplatin, etoposide (R-ICE); bendamustine-R; lenalidomide; and an oral regimen of etoposide, procarbazine, and cyclophosphamide.

On initial examination, the patient's visual acuity was $20 / 30$ $\mathrm{OU}$ and visual fields were full to confrontation. Intraocular pressure was within normal limits (OD: $12 \mathrm{~mm} \mathrm{Hg}$, OS: $18 \mathrm{~mm} \mathrm{Hg}$ ) but relatively elevated in the left eye. The right eye demonstrated full motility; however, motility of the left eye was noted to be restricted in all gazes, most notably in supraduction ( -1 adduction, -1 abduction, -4 supraduction, -1 infraduction). External examination of the left eye demonstrated proptosis (Hertel exophthalmometry: $17 \mathrm{~mm} \mathrm{OD}, 25 \mathrm{~mm}$ OS), resistance to retropulsion, and mild tenderness to palpation (Fig. 1a). Slit lamp and dilated fundus examination was noncontributory. CT images of the orbits without contrast at the time of presentation (Fig. 1b-e) demonstrated a new intraorbital mass that did not have bony involvement, displaced the extraocular muscles inferiorly and medially, and was presumed to be MCL. In consultation with the oncology service, the patient was discharged on $60 \mathrm{mg}$ prednisone every day (for 10 days) and scheduled for additional evaluation by the oculoplastics and oncology services as an outpatient to address the need for possible biopsy or changes in his overall treatment plan. 


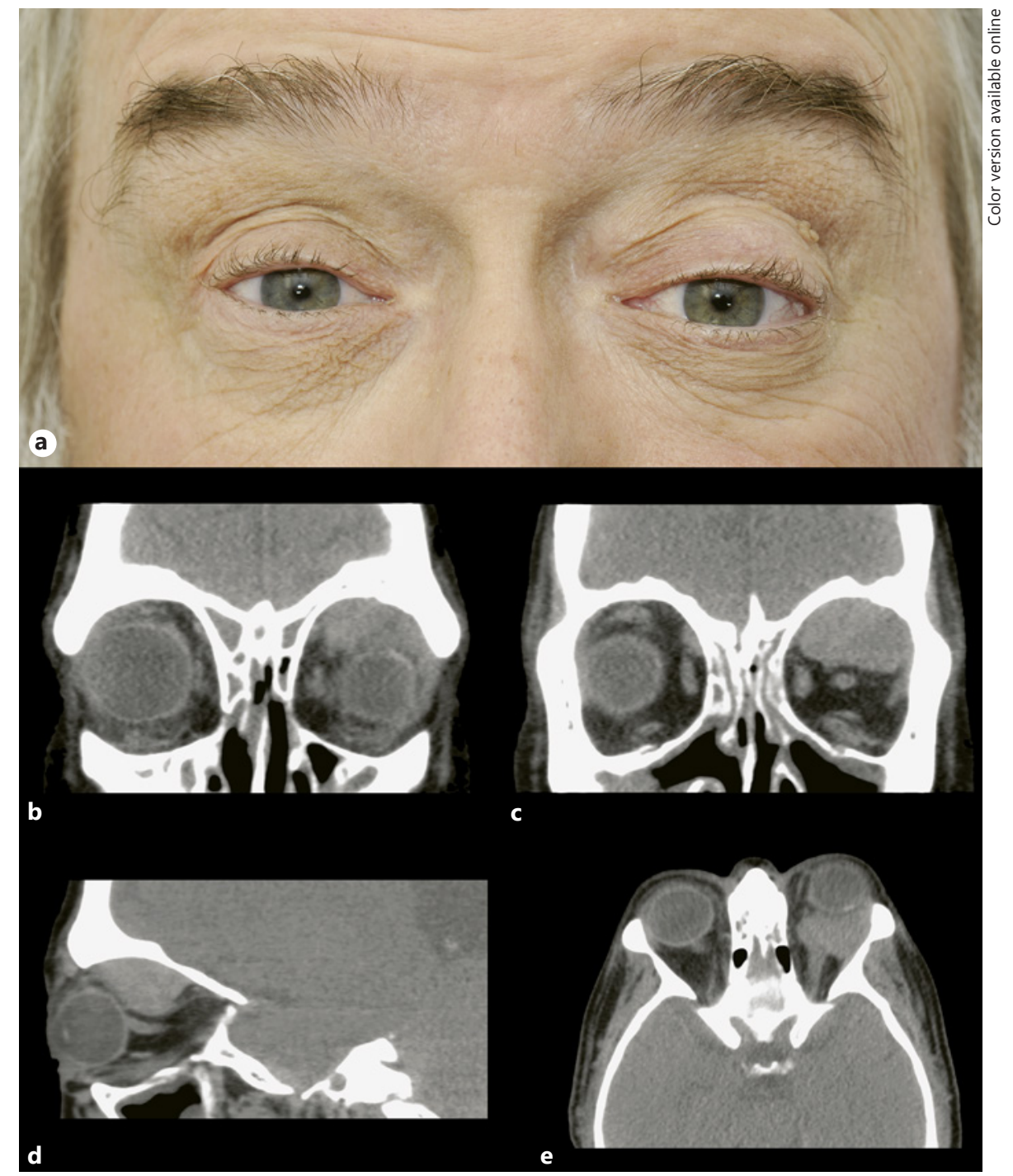

Fig. 1. a Photograph of the patient at the time of presentation showing left-sided proptosis. b-e CT imaging of the orbits without contrast demonstrating orbital mass; coronal (b, c), sagittal (d), and transverse $(\mathbf{e})$ sections are shown.

At outpatient follow-up, 4 days after initial presentation, the patient reported improvement on prednisone therapy and reported only occasional dull left eye pain and intermittent diplopia. At the time of this visit, there was no evidence of optic nerve compromise and biopsy of the mass was initially deferred although reconsidered to rule out central nervous system (CNS) involvement in order for the patient to be eligible for a clinical trial of ibrutinib. Additional images obtained by MRI on the day of biopsy prior to procedure revealed that the mass had not appreciably changed in size since the initial CT study. A diagnostic biopsy was subsequently performed and $2-3 \mathrm{~cm}^{3}$ of tissue was obtained. Cerebrospinal fluid was also obtained the following day by lumbar puncture and revealed no abnormal cells.

Biopsy of the orbital mass showed two discrete CD5-positive abnormal B-cell populations, one of which consisted of small lymphocytes immunophenotypically similar to the previously diagnosed MCL, as well as a distinct component of larger cells (Fig. 2a). Morphologically, the small cell component was composed of mo- notonous small lymphocytes with round or occasionally notched nuclei, condensed chromatin, indistinct nucleoli, and scant cytoplasm. The large cell component was composed of medium to large-sized atypical cells, with slightly irregular or notched nuclear contours, open chromatin, occasional small distinct nucleoli, and small to moderate amounts of cytoplasm. Immunohistochemistry demonstrated the small cell component to be cyclin D1 positive and MUM-1 negative whereas the large cell component was cyclin D1 negative and MUM-1 positive (Fig. 2d). The large cell component also had a markedly elevated Ki-67 proliferative index (Ki-67 staining $\sim 80 \%$ of the large cell component and $<5 \%$ of the small cell component; Fig. 2e). Follow-up immunohistochemistry demonstrated TP53 and SOX11 positivity in the large cell component (the small cell component was not able to be assessed due to insufficient tissue from the small cell component remaining in the sample). Flow cytometry demonstrated the following phenotype for the monotypic B cells: CD5 positive, CD20 positive, CD23 negative, CD11c negative, CD10 negative, and CD200 negative (see also 
Fig. 2. a Hematoxylin and eosin stain of orbital biopsy showing the small cell component (predominantly right side) and the large cell component (predominantly left side) that exhibited blastic transformation. b Immunohistochemistry demonstrates that both small cell and large cell components of the lymphoma stained positive for CD20. c The small cell component showed strong staining for CD5 whereas the large cell component showed weak CD5 staining. $\mathbf{d}$ Staining for cyclin D1 was positive in the small cell component and negative in the large cell component. e Ki-67 staining was mostly negative in the small cell component and mostly positive in the large cell component.

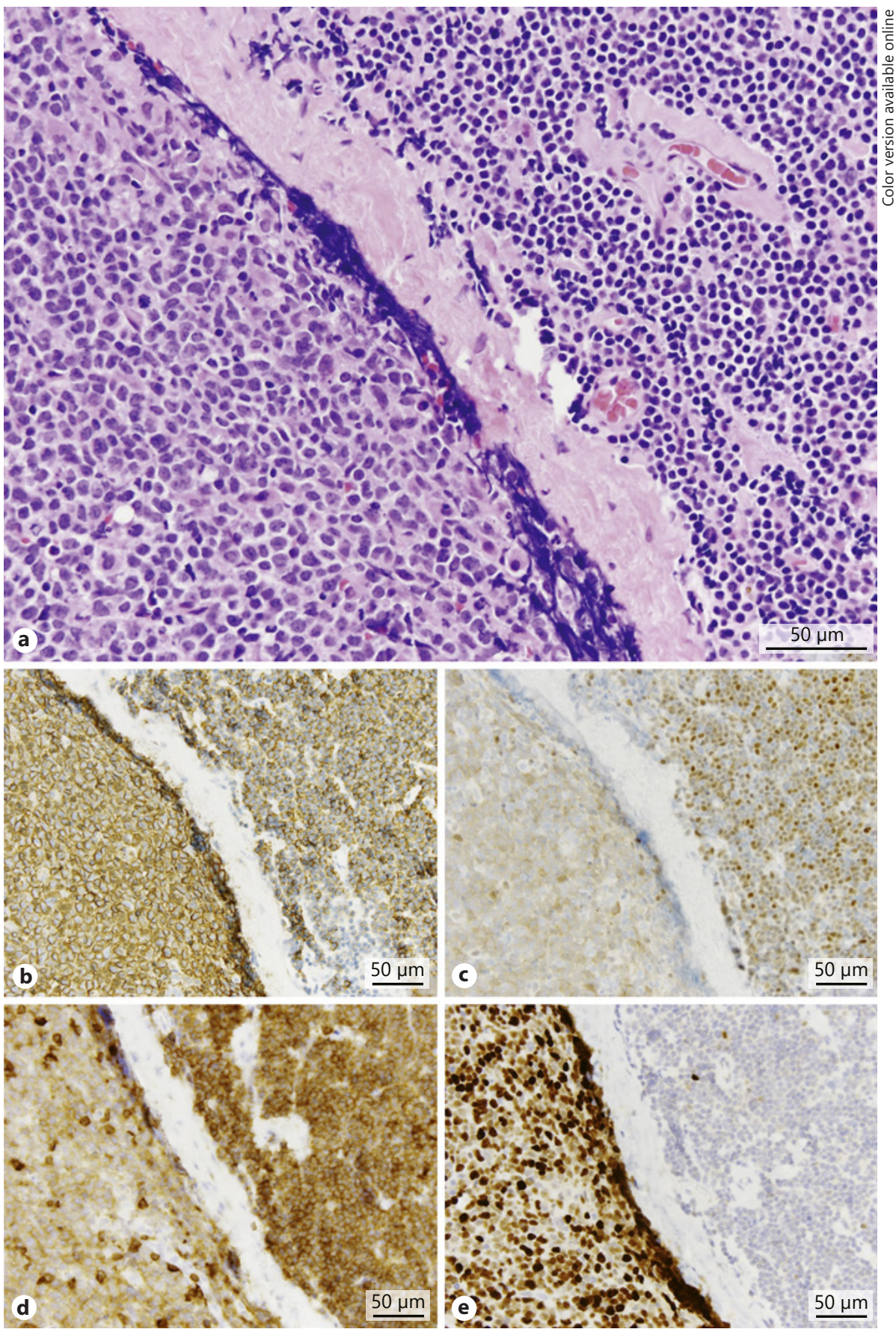

Fig. $2 \mathrm{~b}$ and $\mathrm{c}$ for immunohistochemistry for CD20 and CD5). FISH studies revealed rearrangements between CCND1 and IGH genes, representative of the $t(11 ; 14)$ translocation, in a majority of cells $(89.5 \%)$ as well as an extra copy of BCL6 in some of the cells (36.5\%); these findings were observed in both the small cell and large cell components of the orbital biopsy specimen. Subsequent IGH rearrangement studies performed separately on the small cell and large cell components of the orbital biopsy specimen showed that they were each derived from identical clonal populations. A clone with an $I G H$ product of identical size was also detected in a bone marrow specimen collected $\sim 12$ years prior to this presentation (Fig. 3). Collectively, these findings suggested that the classical component of the MCL exhibited blastic transformation to a more aggressive variant of MCL.

In the weeks following the biopsy, the orbital involvement of the lymphoma progressed, and the patient received palliative radiation therapy to the left orbit with symptomatic improvement following treatment. PET scan at the onset of radiation treatment 


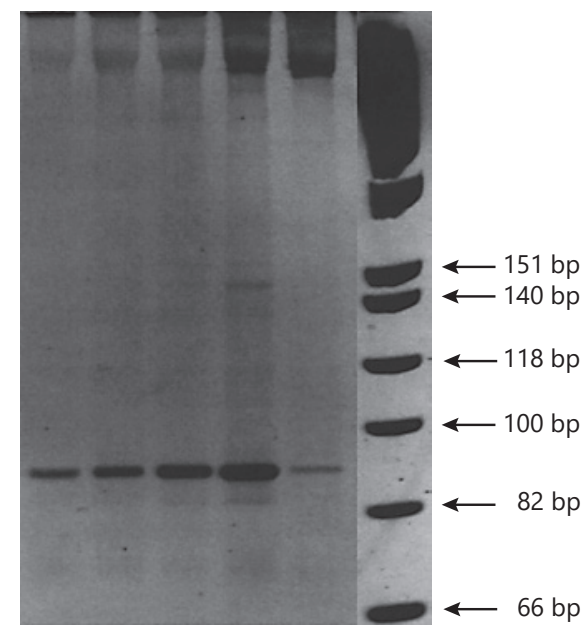

Fig. 3. Immunoglobulin heavy chain gene rearrangement studies were performed using samples from the small cell and large cell portions obtained by biopsy of the orbital mass. DNA was amplified by PCR using consensus primer for the variable framework III and the joining region of the immunoglobulin heavy chain (IGH) locus on chromosome 14q23. Morphologically distinct small cell and large cell components obtained from an orbital biopsy sample are shown in duplicate in lanes 1 and 2 and 3 and 4 (starting from left). Sample derived from bone marrow biopsy nearly 12 years prior to biopsy of the orbital mass is shown in lane 5 . The presence of a single band indicates that the small cell and large cell components of the orbital biopsy are of identical clonal populations and that the samples from the orbital biopsy and the earlier bone marrow biopsy are also from a common clonal population.

showed only an FDG-avid mass within the upper aspect of the left orbit compatible with active lymphoma. Approximately 4 months following the patient's initial presentation, the patient experienced further recurrence of orbital involvement with proptosis presumed to be the lymphoma; he received additional treatment with radiation therapy for the enlargement of the orbital mass. The patient died 8 months following initial presentation.

\section{Discussion}

MCL represents a small portion of cases of lymphoma affecting the orbit and ocular adnexa. Approximately $1-9 \%$ of cases of lymphoma reported to affect the orbital and ocular structures are attributed to MCL [15-17]. Like MCL in general, MCL in the orbit and ocular adnexa similarly tends to present predominantly in males $[15,18,19]$ and the age at presentation typically occurs in individuals aged $>60$ years, with a median age of presentation often reported to be in the early 70s (which is slightly older than MCL in general although ocular adnexal MCL may occur over a broad age range, including patients in their early 30 s) $[15,19]$. In the case we have described, the patient was male and presented with ocular signs and symptoms at age 65 , although this occurred 24 years following the patient's initial diagnosis of MCL. The prognosis may be similar for all patients with MCL at the time of orbital and ocular adnexal MCL involvement. A recent report suggests that 5-year disease-specific survival rates among patients with primary orbital and ocular adnexal MCL, systemic MCL with involvement of the orbit and ocular adnexa, and relapse of systemic MCL in the orbital and ocular adnexal region do not differ (5-year disease-specific survival rate of $38 \%$ ) [18].

The orbit is the most frequently reported site of MCL involvement in the orbit and ocular adnexa $[15,18,19]$, with variable reported involvement of the eyelids, conjunctiva, and lacrimal gland and infrequent involvement of the lacrimal sac $[15,18,19]$. Clinically, the most commonly reported signs of MCL in these regions are related to the mass effect of the tumor, with proptosis or the presence of an eyelid or conjunctival mass often reported [15, $18,19]$. Less frequently observed clinical signs may also include ptosis, chemosis, and the limitation of extraocular movements $[15,18,19]$. Pain, irritation, epiphora, and swelling are the most frequently reported symptoms of orbital and ocular adenexal MCL $[15,18]$, while decreased visual acuity and diplopia are less frequently reported symptoms $[15,18,19]$. In our case, the patient presented with features related to the mass effect of the orbital tumor, notably proptosis and resistance to retropulsion, but also in association with less frequently reported signs and symptoms including diplopia, limitation of extraocular movements, and decreased visual acuity.

Unique to this case is the transformation of classical MCL to the blastoid variant of MCL that was observed within the orbit. The blastoid variant of MCL is only reported in a small proportion of cases of MCL $[3,12]$ and it generally has a poor prognosis $[3,12]$. Only two reports have identified the blastoid form of MCL in the orbital and ocular adnexal region [20]. While the blastoid variant of MCL may occur de novo or following transformation of MCL [11, 13], reports of MCL exhibiting transformation to the blastoid variant of MCL are rare $[21,22]$.

Various markers may be indicative of the aggressiveness of MCL and overall prognosis. Evidence suggests that the Ki-67 index is useful for predicting overall prognosis with MCL, with higher Ki-67 indices associated with a poorer overall prognosis [23]. In an analysis of the 
Ki-67 index for blastoid type MCL (combining blastic and pleomorphic variants), a Ki-67 index of $<30 \%$ was associated with better overall survival than those with a Ki-67 index of $\geq 30 \%$, suggesting that prognosis, even for blastoid type MCL may be explained by the Ki-67 index [24]. In the case presented, the patient was observed to have a high Ki-67 index in the blastoid component and low Ki-67 in the classical MCL component of biopsied orbital tumor. Additionally, the blastoid component demonstrated was strongly positive for TP53. Strong expression of TP53 is also associated with more aggressive presentations of MCL [25]. We also report in this case SOX11 positivity in the blastoid component of the MCL. While the presence of SOX11 expression has been suggested to be helpful in the identification of MCL [10], the presence of SOX11 expression has been reported to be associated with both MCL exhibiting a more indolent course as well as poor outcomes [26].

Although the prognosis for orbital and ocular adnexal MCL has been historically poor, it may be improving due to the use of monoclonal antibodies and the activity of novel agents, including ibrutinib, lenalidomide, and venetoclax. A recent retrospective multicenter study of patients with ocular adnexal MCL from 1980 to 2015 reported that survival may be improved in patients receiving rituximab in addition to chemotherapy [18]. In this case, the patient did receive rituximab in combination with numerous other chemotherapy treatments as part of his treatment.

In regard to the blastoid variant of MCL, the optimal approach to treatment has not been determined [20,27]. As the blastoid variant of MCL is aggressive, more intensive therapy should be considered. Favorable responses for those with blastoid cytology has been reported in a study of an intense chemoimmunotherapy regimen of rituximab in combination with fractionated cyclophosphamide, vin- cristine, doxorubicin, and dexamethasone (R-HyperCVAD) alternating with rituximab in combination with high-dose methotrexate-cytarabine (R-MA) [28]. Blastoid histology is associated with a greater risk of involvement of the CNS $[29,30]$ and cerebrospinal fluid analysis and neuroimaging may be considered in patients with blastoid histology [1]. For patients with blastoid morphology, the use of empirical CNS-penetrating chemotherapy has been suggested to be possibly beneficial, although there is no clear consensus regarding CNS prophylaxis [27].

In this case, we describe the case of a rarely observed transformation of MCL from classical MCL to the blastoid variant of MCL. Clinically, the possibility of transformation of MCL may indicate the potential utility of tumor biopsy if transformation is expected or the MCL appears to have become more aggressive.

\section{Statement of Ethics}

Our institutional review board reviewed the case and granted exempt status for the case report.

\section{Disclosure Statement}

The authors have no conflicts of interest to disclose.

\section{Funding Sources}

The study was supported by a Research to Prevent Blindness unrestricted departmental award (Vanderbilt University Medical Center, Department of Ophthalmology and Visual Sciences). M.A. De Niear was supported by the NIGMS of the National Institutes of Health under award number T32GM007347. The content is solely the responsibility of the authors and does not necessarily represent the official views of the National Institutes of Health.

\section{References}

1 Cheah CY, Seymour JF, Wang ML. Mantle cell lymphoma. J Clin Oncol. 2016 Apr; 34(11):1256-69.

2 Fu S, Wang M, Lairson DR, Li R, Zhao B, Du $\mathrm{XL}$. Trends and variations in mantle cell lymphoma incidence from 1995 to 2013: A comparative study between Texas and National SEER areas. Oncotarget. 2017 Nov; 8(68): 112516-29.

3 Bosch F, López-Guillermo A, Campo E, Ribera JM, Conde E, Piris MA, et al. Mantle cell lymphoma: presenting features, response to therapy, and prognostic factors. Cancer. 1998 Feb;82(3):567-75.
4 Vose JM. Mantle cell lymphoma: 2017 update on diagnosis, risk-stratification, and clinical management. Am J Hematol. 2017 Aug;92(8): 806-13.

5 Andersen NS, Jensen MK, de Nully Brown P, Geisler CH. A Danish population-based analysis of 105 mantle cell lymphoma patients: incidences, clinical features, response, survival and prognostic factors. Eur J Cancer. 2002 Feb;38(3):401-8. 
6 Tiemann M, Schrader C, Klapper W, Dreyling $\mathrm{MH}$, Campo E, Norton A, et al.; European MCL Network. Histopathology, cell proliferation indices and clinical outcome in 304 patients with mantle cell lymphoma (MCL): a clinicopathological study from the European MCL Network. Br J Haematol. 2005 Oct;131(1):29-38.

7 Jares P, Colomer D, Campo E. Genetic and molecular pathogenesis of mantle cell lymphoma: perspectives for new targeted therapeutics. Nat Rev Cancer. 2007 Oct;7(10):75062.

8 Argatoff LH, Connors JM, Klasa RJ, Horsman DE, Gascoyne RD. Mantle cell lymphoma: a clinicopathologic study of 80 cases. Blood. 1997 Mar;89(6):2067-78.

9 Barista I, Romaguera JE, Cabanillas F. Mantle-cell lymphoma. Lancet Oncol. 2001 Mar; 2(3):141-8.

10 Mozos A, Royo C, Hartmann E, De Jong D, Baró C, Valera A, Fu K, Weisenburger DD, Delabie J, Chuang S-S: SOX11 expression is highly specific for mantle cell lymphoma and identifies the cyclin D1-negative subtype. Haematologica. 2009 Nov;94(11):1555-62.

11 László T, Matolcsy A. Blastic transformation of mantle cell lymphoma: genetic evidence for a clonal link between the two stages of the tumour. Histopathology. 1999 Oct;35(4):355-9.

12 Bernard M, Gressin R, Lefrère F, Drénou B, Branger B, Caulet-Maugendre S, et al. Blastic variant of mantle cell lymphoma: a rare but highly aggressive subtype. Leukemia. 2001 Nov;15(11):1785-91.

13 Nakatsuka SI, Nagatomo T, Nagano T, Goto T, Hashimoto K. Classical type and blastoid variant mantle cell lymphoma in the same lymph node: histology and cytological findings from a touch imprint specimen. Diagn Cytopathol. 2017 Apr;45(4):364-70.
14 Pérez-Galán P, Dreyling M, Wiestner A. Mantle cell lymphoma: biology, pathogenesis, and the molecular basis of treatment in the genomic era. Blood. 2011 Jan;117(1):26-38.

15 Rasmussen P, Sjö LD, Prause JU, Ralfkiaer E, Heegaard S. Mantle cell lymphoma in the orbital and adnexal region. Br J Ophthalmol. 2009 Aug;93(8):1047-51.

16 Rootman DB, Mavrikakis I, Connors JM, Rootman J. Primary, unilateral ocular adnexal lymphoma: disease progression and longterm survival. Ophthal Plast Reconstr Surg. 2011 Nov-Dec;27(6):405-9.

17 Ferry JA, Fung CY, Zukerberg L, Lucarelli MJ, Hasseriian RP, Preffer FI, et al. Lymphoma of the ocular adnexa: A study of 353 cases. Am J Surg Pathol. 2007 Feb;31(2):170-84.

18 Knudsen MK, Rasmussen PK, Coupland SE, Esmaeli B, Finger PT, Graue GF, et al. Clinicopathological Features of Ocular Adnexal Mantle-Cell Lymphoma in an International Multicenter Cohort. JAMA Ophthalmol. 2017 Dec;135(12):1367-74.

19 Looi A, Gascoyne RD, Chhanabhai M, Connors JM, Rootman J, White VA. Mantle cell lymphoma in the ocular adnexal region. Ophthalmology. 2005 Jan;112(1):114-9.

20 Rašić DM, Knežević M, Terzić T, Vlajković G: Bilateral ocular panadnexal mass as initial presentation of systemic blastoid variant of mantle-cell lymphoma. Surv Ophthalmol. 2017 Jan-Feb;62(1):83-88.

21 Kaleem Z, Wakoff AR, Smith RP, Hess JL. Blastic transformation of mantle cell lymphoma. Arch Pathol Lab Med. 1996 Jun;120(6): 577-80.

22 Kasamon YL, Burns KH. Blastic transformation of mantle cell lymphoma. Blood. 2012 Sep;120(12):2359-2359.

23 Katzenberger T, Petzoldt C, Höller S, Mäder U, Kalla J, Adam P, et al. The Ki67 proliferation index is a quantitative indicator of clinical risk in mantle cell lymphoma. Blood. 2006 Apr;107(8):3407-3407.
24 Hoster E, Rosenwald A, Berger F, Bernd HW, Hartmann S, Loddenkemper C, et al. Prognostic value of Ki-67 index, cytology, and growth pattern in mantle-cell lymphoma: results from randomized trials of the European mantle cell lymphoma network. J Clin Oncol. 2016 Apr;34(12):1386-94.

25 Nordström L, Sernbo S, Eden P, Grønbaek K, Kolstad A, Räty R, et al. SOX11 and TP53 add prognostic information to MIPI in a homogenously treated cohort of mantle cell lymphoma - a Nordic Lymphoma Group study. Br J Haematol. 2014 Jul;166(1):98-108.

26 Beà S, Amador V. Role of SOX11 and genetic events cooperating with cyclin D1 in mantle cell lymphoma. Curr Oncol Rep. 2017 Jun; 19(6):43.

27 McKay P, Leach M, Jackson B, Robinson S, Rule S. Guideline for the management of mantle cell lymphoma. Br J Haematol. 2018 Jul;182(1):46-62.

28 Romaguera JE, Fayad LE, Feng L, Hartig K, Weaver P, Rodriguez MA, et al. Ten-year follow-up after intense chemoimmunotherapy with Rituximab-HyperCVAD alternating with Rituximab-high dose methotrexate/cytarabine (R-MA) and without stem cell transplantation in patients with untreated aggressive mantle cell lymphoma. Br J Haematol. 2010 Jul;150(2):200-8.

29 Cheah CY, George A, Giné E, Chiappella A, Kluin-Nelemans HC, Jurczak W, et al.; European Mantle Cell Lymphoma Network. Central nervous system involvement in mantle cell lymphoma: clinical features, prognostic factors and outcomes from the European Mantle Cell Lymphoma Network. Ann Oncol. 2013 Aug;24(8):2119-23.

30 Ferrer A, Bosch F, Villamor N, Rozman M, Graus F, Gutiérrez G, et al. Central nervous system involvement in mantle cell lymphoma. Ann Oncol. 2008 Jan;19(1):135-41. 